\title{
Terahertz Technology and Its Biomedical Application
}

\author{
Guoli Wu1, Haizheng Gao1, Yuan Wang2, Yikai Jin², Yingwei Li1* ${ }^{*}$ Chengbiao Lu' ${ }^{2,3 *}$ \\ ${ }^{1}$ College of Information Science and Engineering, Yanshan University, Qinhuangdao, China \\ ${ }^{2}$ Basic Medical College, Xinxiang Medical College, Xinxiang, China \\ ${ }^{3}$ College of Medicine, Yangtze University, Wuhan, China \\ Email:982674981@qq.com,1790122039@qq.com, 465156599@qq.com,2839599171@qq.com, *lyw@ysu.edu.cn, \\ ^2331202185@qq.com
}

How to cite this paper: Wu, G.L., Gao, H.Z., Wang, Y., Jin, Y.K., Li, Y.W. and Lu, C.B. (2019) Terahertz Technology and Its Biomedical Application. Yangtze Medicine, 3, 157-162.

https://doi.org/10.4236/ym.2019.33016

Received: January 4, 2019

Accepted: June 25, 2019

Published: June 28, 2019

Copyright $\odot 2019$ by author(s) and Scientific Research Publishing Inc. This work is licensed under the Creative Commons Attribution International License (CC BY 4.0).

http://creativecommons.org/licenses/by/4.0/

\begin{abstract}
The terahertz wave is an electromagnetic wave with a spectrum ranged between the microwave and the infrared. Some studies found that terahertz had the advantages of low energy, non-invasive, high resolution and penetration. Based on these advantages, $\mathrm{THz}$ is relatively safe for biomolecules and it has been applied in terahertz imaging. So we summarized these reports in this mini-review.
\end{abstract}

\section{Keywords}

Terahertz, THz Biomedical Interaction, THz Imaging

\section{Introduction}

The terahertz wave in general term is a small electromagnetic wave between the microwave and the infrared in the electromagnetic spectrum [1], as shown in Figure 1. Terahertz frequency band is the only area with insufficient research in the whole electromagnetic frequency range [2]. With the development of electronic and optical technologies, terahertz source and detection technology have made creative breakthroughs, resulting in the rapid development of $\mathrm{THz}$ technology. At present, THz technology has been used in many scientific researches and application fields such as military, security inspection, biomedical health [3].

As the photon energy of $\mathrm{THz}$ waves is only one in a million of that of X-rays [4] [5], the THz does not cause chemical or physical damage to biological systems. One of the advantages for $\mathrm{THz}$ is safe in application. $\mathrm{THz}$ waves have very high spectral resolution for macromolecules [6] and can be used for detection of biomacromolecule conformation, which is difficult to detect by conventional 


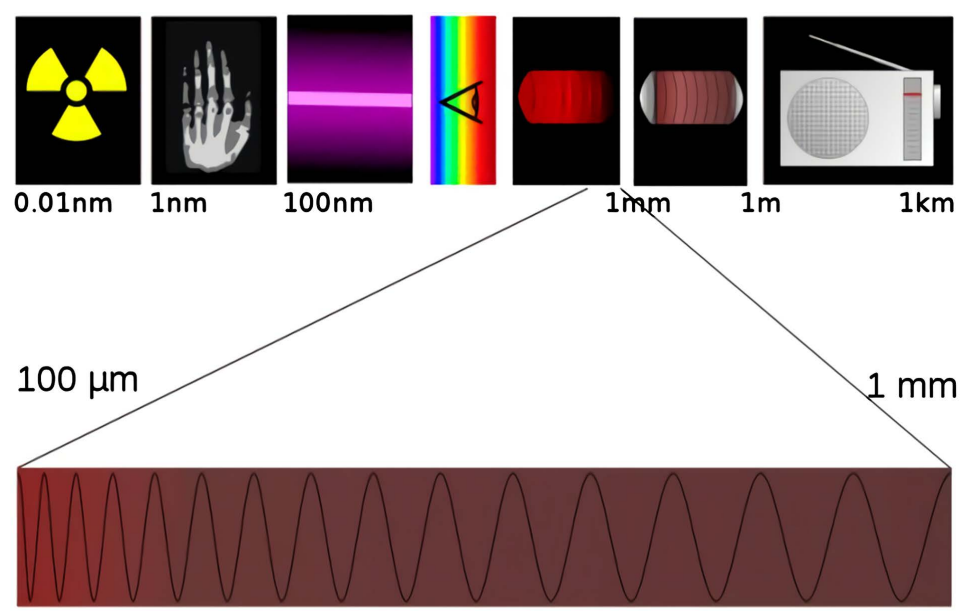

Figure 1. Terahertz band.

detection methods. $\mathrm{THz}$ wave has strong penetration [7], due to the interference and diffraction ability, it interacts very strongly with polar molecules, thus it is better in biological tissue imaging than other electromagnetic waves such as $\mathrm{X}$-rays. The THz wave has very good coherence [8]. The parameters of the object radiated by $\mathrm{THz}$ such as dielectric constant and refractive index, can be obtained more accurately [9], as shown in Figure 2.

\section{The Biological Effect of Terahertz}

In terms of the $\mathrm{THz}$ influence on biomolecules, the vibrational levels of many biomolecules such as sugars, proteins, DNA, RNA are in the range of $\mathrm{THz}$ wave band. When $\mathrm{THz}$ waves are radiated onto biomolecules or organisms, the high-intensity absorption of $\mathrm{THz}$ by biomolecules alter the biomolecule's conformation and function, which may have a series of unpredictable effects on organisms. Therefore, we summarized several studies regarding the effects of $\mathrm{THz}$ wave on biological molecules.

Recent study by Turton et al. showed that $\mathrm{THz}$ vibrational motion affects efficiency of protein-ligand binding and alters function of protein molecules [10]. Changes in protein conformation can also be caused by low-frequency intramolecular [11]. Brandt et al. used $\mathrm{THz}$ wave to detect the interaction in proteins to predict the changes of protein function [12]. Olshevskaya et al. found that long term exposed to $\mathrm{THz}$ (40 - 50 hours) caused the morphological abnormalities of the cell membranes and intracellular structures [13]. By using a $0.1 \mathrm{THz}$ continuous laser to irradiate the separated lymphocytes and Korenstein et al. found that all stains of cells showed an unsynchronized replication after $24 \mathrm{~h}$ of radiation. The authors believe that these phenomena are caused by $\mathrm{THz}$ induction of instability DNA molecules [14]. Kulipanovt et al. found that high-power $\mathrm{THz}$ radiation $(2.3 \mathrm{THz})$ can destroy DNA double-stranded hybridization and disrupt hydrogen bonds between target DNA double-strands in the absence of free water [15]. In addition, Alexandrov et al. studied the response of mouse stem cells to $\mathrm{THz}$ waves and found that the transcriptional activity of thermo-sensitive genes 
were increased [16]. Researchers at Ruhr University in Germany clearly observed the interaction between water molecules and proteins using $\mathrm{THz}$ waves [17]. Edwards et al. have also demonstrated that $\mathrm{THz}$ mainly interacts with hydrogen bonds in biomolecules [18] [19]. Study about THz absorption peaks of human serum demonstrated that the $\mathrm{THz}$ absorption peak of protein thermal denaturation is of a reversal characteristic, which reflected the dynamic change of serum albumin hydration structure [20]. The above experiments demonstrate that non-ionizing electric field of $\mathrm{THz}$ waves influences the function of organisms by a non-thermal mechanism, which is quite different from the mechanism by which high-energy rays such as UV and X-ray. This kind of nonlinear resonant mechanism often appears in THz stimulation [21] [22]. Conformational changes resulting from this mechanism can subsequently interfere with protein transfer and binding processes, thus profoundly impacts on gene expression in cells. Research has found that terahertz radiation destroys the barrier properties of the membrane and this process is reversible, so terahertz can be an inducer for the transmission of bioactive compounds [23]. BOROVKOVA has found that terahertz radiation increases the number of apoptotic cells, they believe that the diagnostic application of $\mathrm{THz}$ radiation may be limited by radiation power density and irradiation time [24].

Taken together, $\mathrm{THz}$ affects biomolecules only under a condition of high power $(>1 \mathrm{THz})$ and long time (>24 h), so THz is relatively safe for biomolecules.

\section{Terahertz Imaging}

$\mathrm{THz}$ imaging is one of the most promising methods in biomedical science. $\mathrm{THz}$ time-domain spectroscopy has been applied to biological macromolecules detection, cancer research, determination of burn severity. THz waves are sensitive to polar molecules such as water molecules in biomolecules, thus $\mathrm{THz}$ imaging of biological tissues has more advantageous than other ways. THz imaging is of high spatial resolution, which reaches several tens of micrometers. Its penetration ability is also very strong, which can be used for tomography of biological systems. Started by $\mathrm{Hu}$ [25], Fitzgerald et al. [26] tested the THz refractive index and absorptivity of human blood, muscle, skin, fat, vein and even nerve tissue. The results showed that $\mathrm{THz}$ had a good identification for different tissues, and confirmed the feasibility of its clinical application. The study of THz imaging on 22 isolated breast cancer patients [27] showed that a high correlation in breast cancer detection between pathological examination microscopic image and $\mathrm{THz}$ image. The study of $\mathrm{THz}$ imaging on brain tissue demonstrated that $\mathrm{THz}$ wave spectrum can distinguish between gray matter (neuron body) and white matter (nerve axon) and that there are reflection spectrum differences between gray matter and white matter. Seung Jae Oh obtained the terahertz images of fresh brains with and without tumor by using reflection type terahertz pulses imaging system [28], as shown in Figure 3. From terahertz images of different biological specimens, the water content and dehydration characteristics of fat 


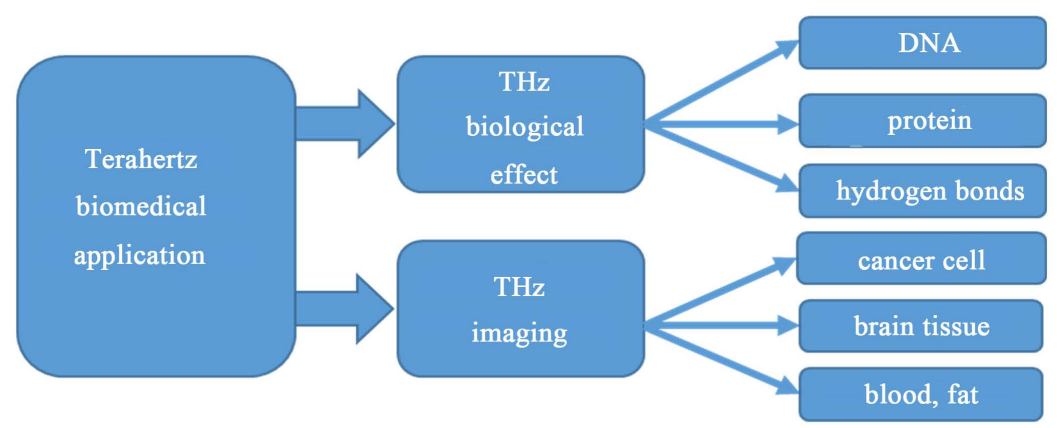

Figure 2. Terahertz research direction.

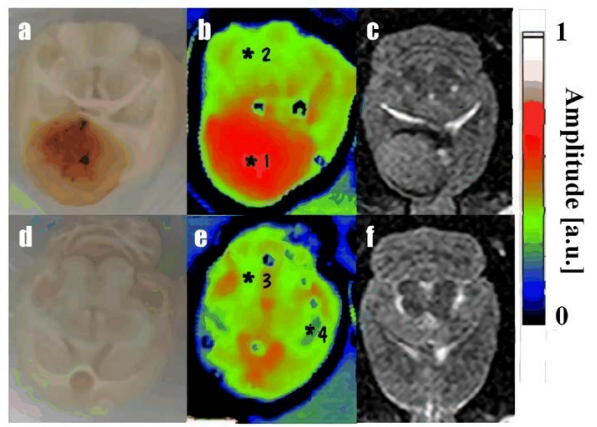

Figure 3. Terahertz pulses imaging system.

and muscle tissue are accurately distinguished and the results show that terahertz imaging technology provides a valuable measuring platform for biological sensing [29]. Zhang $M$ found that terahertz can be combined with nanotechnology to help improve terahertz biometric imaging capabilities and provide advanced imaging results [30]. The research of terahertz imaging have shown that the amplitude of reflected signal in severely scalded tissues was lower than that in normal tissues, so the terahertz may be used to detect scald wounds.

\section{Conclusion}

Based on the low energy, non-invasive, high resolution and penetration, it is foreseeable that $\mathrm{THz}$ will be widely applied in biomolecules detection and clinical imaging.

\section{Conflicts of Interest}

The authors declare no conflicts of interest regarding the publication of this paper.

\section{References}

[1] Walther, M., Fischer, B.M., Ortner, A., et al. (2010) Chemical Sensing and Imaging with Pulsed THz Radiation. Analytical and Bioanalytical Chemistry, 397, 1009-1017. https://doi.org/10.1007/s00216-010-3672-1

[2] Wei, H. (2016) Millimeter Wave and Terahertz Technology. Scientia Sinica, 46, 1086-1107. 
[3] Yang, P.F., Yao, J.Q., Bing, P.B., et al. (2011) Source and Characteristics of THz Wave. Laser \& Infrared, 41, 125-126.

[4] Pickwell, E. and Wallace, V.P. (2006) Biomedical Applications of THz Technology. Journal of Physics D: Applied Physics, 39, R301. https://doi.org/10.1088/0022-3727/39/17/R01

[5] Plusquellic, D.F., Demaison, J. and Ilyushin, V.V. (2006) The Microwave Spectrum of a Two-Top Peptide Mimetic: The N-Acetyl Alanine Methyl Ester Molecule. The Journal of Chemical Physics, 125, Article ID: 104312. https://doi.org/10.1063/1.2348871

[6] Yamamoto, K., Tominaga, K., Sasakaw, H., et al. (2005) THz Time-Domain Spectroscopy of Amino Acids and Polypeptides. Biophysical Journal, 89, 22-24. https://doi.org/10.1529/biophysj.105.067447

[7] Balu, R., Zhang, H., Zukowski, E., Chen, J.-Y., Markelz, A.G. and Gregurick, S.K. (2008) $\mathrm{THz}$ Spectroscopy of Bacteriorhodopsin and Rhodopsin: Similarities and Differences. Biophysical Journal, 94, 3217-3226. https://doi.org/10.1529/biophysj.107.105163

[8] Markelz, A.G., Roitberg, A. and Heilweil, E.J. (2000) Pulsed THz Spectroscopy of DNA, Bovine Serum Albumin and Collagen between 0.1 and $2.0 \mathrm{THz}$. Chemical Physics Letters, 320, 42-48. https://doi.org/10.1016/S0009-2614(00)00227-X

[9] Arora, A., Luong, T.Q., Kruger, M., et al. (2012) THz-Time Domain Spectroscopy for the Detection of PCR Amplified DNA in Aqueous Solution. Analyst, 137, 575-579. https://doi.org/10.1039/C2AN15820E

[10] Turton, D.A., Senn, H.M., Harwood, T., Lapthorn, A.J., Ellis, E.M. \& Wynne, K. (2014) THz Underdamped Vibrational Motion Governs Protein-Ligand Binding in Solution. Nature Communications, 5, 3999. https://doi.org/10.1038/ncomms4999

[11] Cherkasova, O.P., Fedorov, V.I., Nemova, E.F., et al. (2009) Influence of THz Laser Radiation on the Spectral Characteristics and Functional Properties of Albumin. Optics and Spectroscopy, 107, 534-537. https://doi.org/10.1134/S0030400X09100063

[12] Brandt, N.N., Chikisher, A.Y., Mankova, A.A. and Pogodin, A.S. (2012) THz and IR Spectroscopy of Molecular Systems That Simulate Function-Related Structural Changes of Proteins. Spectroscopy-An International Journal, 27, 429-432. https://doi.org/10.1155/2012/745136

[13] Olshevskaya, J.S., Ratushnyak, A.S., Petrov, A.K., Kozlov, A.S. and Zapara, T.A. (2008) Effect of $\mathrm{THz}$ Electromagnetic Waves on Neurons Systems. International Conference on Computational Technologies in Electrical and Electronics Engineering, Novosibirsk, 21-25 July 2008, 210-211.

https://doi.org/10.1109/SIBIRCON.2008.4602607

[14] Korenstein-Ilan, A., Barbul, A., Hasin, P., Eliran, A., Gover, A. and Korenstein, R. (2008) THz Radiation Increases Genomic Instability in Human Lymphocytes. Radiation Research, 170, 224-234. https://doi.org/10.1667/RR0944.1

[15] Kulipanov, G.N., Gavrilov, N.G., Knyazev, B.A., et al. (2008) Research Highlights from the Novosibirsk 400W Average Power THz FEL. Terahertz Science \& Technology, 1, 107-125.

[16] Alexandrov, B.S., Rasmussen, K.O., Bishop, A., et al. (2011) Non-Thermal Effects of $\mathrm{THz}$ Radiation on Gene Expression in Mouse Stem Cells. Biomedical Optics EXpress, 2, 2679-2689. https://doi.org/10.1364/BOE.2.002679

[17] Ebbinghaus, S., Kim, S.J., Heyden, M., et al. (2007) An Extended Dynamical Hydration Shell around Proteins. Proceedings of the National Academy o Sciences of the 
USA, 104, 20749-20752. https://doi.org/10.1073/pnas.0709207104

[18] Edwards, W.F., Young, D.D. and Deiters, A. (2009) The Effect of Microwave Irradiation on DNA Hybridization. Organic \& Biomolecular Chemistry, 7, 2506-2508. https://doi.org/10.1039/b903609a

[19] Fischer, B.M., Walther, M. and Uhd Jepsen, P. (2002) Far-Infrared Vibrational Modes of DNA Components Studied by THz Time-Domain Spectroscopy. Physics in Medicine \& Biology, 47, 3807-3814. https://doi.org/10.1088/0031-9155/47/21/319

[20] Luong, T.Q., Verina, P.K., Mitra, R., et al. (2011) Do Hydration Dynamics Follow the Structural Perturbation during Thermal Denaturation of a Protein: A THz Absorption Study. Biophysical Journal, 101, 925-933.

https://doi.org/10.1016/j.bpj.2011.05.011

[21] Alexandrov, B.S., Gelev, V., Bishop, A.R., et al. (2010) DNA Breathing Dynamics in the Presence of a THz Field. Physics Letters A, 374, 1214-1217.

https://doi.org/10.1016/j.physleta.2009.12.077

[22] Maniadis, P., Alexandrov, B.S., Bishop, A.R., et al. (2011) Feigenbaum Cascade of Discrete Breathers in a Model of DNA. Physical Review E, Statistical, Nonlinear, and Soft Matter Physics, 83, Article ID: 011904. https://doi.org/10.1103/PhysRevE.83.011904

[23] Zapara, T.A., Treskova, S.P. and Ratushniak, A.S. (2015) Effect of Antioxidants on the Interaction of Terahertz (Submillimeter) Laser Radiation and Neuronal Membrane. Journal of Surface Investigation. X-Ray, Synchrotron and Neutron Techniques, 9, 869-871. https://doi.org/10.1134/S1027451015050195

[24] Borovkova, M., Serebriakova, M., Fedorov, V., et al. (2017) Investigation of Terahertz Radiation Influence on Rat Glial Cells. Biomedical Optics Express, 8, 273-280. https://doi.org/10.1364/BOE.8.000273

[25] Hu, B.B. and Nuss, M.C. (1995) Imaging with THz Waves. Optics Letters, 20, 1716-1718. https://doi.org/10.1364/OL.20.001716

[26] Fitzgerald, A.J., Berry, E., Zinov'ev, N.N., Homer-Vanniasinkam, S., Miles, R.E., et al. (2003) Catalogue of Human Tissue Optical Properties at THz Frequencies. Journal of Biological Physics, 29, 123-128. https://doi.org/10.1023/A:1024428406218

[27] Anthony, J., Wallace, V.P., Jimenez-Linan, M., Bobrow, L., Pye, R.J., et al. (2006) $\mathrm{THz}$ Pulsed Imaging of Human Breast Tumors. Radiology, 239, 533-540. https://doi.org/10.1148/radiol.2392041315

[28] Oh, S.J., Huh, Y.M., Kim, S.H., et al. (2011) THz Pulse Imaging of Fresh Brain Tumor. 36 th International Conference on Infrared, Millimeter and THz Waves, Houston, 2-7 October 2011, 1-2. https://doi.org/10.1109/irmmw-THz.2011.6105230

[29] Guo, L., Wang, X., Han, P., et al. (2017) Observation of Dehydration Dynamics in Biological Tissues with Terahertz Digital Holography. Applied Optics, 56, F173. https://doi.org/10.1364/AO.56.00F173

[30] Zhang, M. and Yeow, J.T.W. (2016) Nanotechnology-Based Terahertz Biological Sensing: A Review of Its Current State and Things to Come. IEEE Nanotechnology Magazine, 10, 30-38. https://doi.org/10.1109/MNANO.2016.2572244 